\title{
CLUSTERS Y PROJECT FINANCE. ANÁLISIS DE SU IMPLEMENTACIÓN EN EL DEPARTAMENTO DE ALTO PARANÁ, PARAGUAY. PERÍODO: 2016-2046
}

\author{
CLUSTERS AND PROJECT FINANCE. ANALYSIS OF ITS IMPLEMENTATION IN \\ THE ALTO PARANA DEPARTMENT, PARAGUAY. PERIOD: 2016-2046
}

\author{
Clara Ruth Riveros González* \\ María Antonella Cabral López*
}

enviado: 25 enero 2018 - aceptado: 12 junio 2018

\begin{abstract}
Resumen
El objetivo de este artículo es identificar los beneficios económicos y sociales de la implementación del Project Finance en un clúster para la producción de stevia en el Departamento de Alto Paraná, Paraguay. Para realizarlo se utilizó el modelo de descuento de flujos de caja y se valoró la realización de este proyecto de inversión. Las aportaciones son, por un lado, que se ofrece un modelo de clúster con Project Finance íntegro, con base en la realidad, que convierte los problemas en oportunidades; por otro lado, se busca generar con la propuesta un mayor desarrollo de este tipo de proyectos innovadores.
\end{abstract}

Código JEL: O16.

Palabras clave: clúster, Project Finance, Paraguay.

\begin{abstract}
The objective of this paper is to identify the economic and social benefits of the implementation of Project Finance in a cluster for the production of stevia in Alto Paraná Department (Paraguay). To execute it, this work designs how to operate the

Riveros González, C. R., \& Cabral López, M. A. (2018). Clusters y Project Finance. Análisis de su implementación en el departamento de Alto Paraná, Paraguay. Período: 2016-2046. Estudios económicos, 35 (71), 73-100.

* Facultad de Ciencias Económicas de la Universidad Nacional de Asunción, Paraguay. Correos electrónicos: ruthriverosg18@gmail.com, antocabral@gmail.com
\end{abstract}


cluster under specific studies and through the Discounted Cash Flow model. The contribution of this paper consists firstable on giving a cluster model with Project Finance based on the reality that turns problems into opportunities and in the other hand, the possibility to generate more interest on the realization of this kind of innovative projects.

JEL Code: O16.

Key words: Cluster, Project Finance, Paraguay.

\section{INTRODUCCIÓN}

A lo largo de los siglos se han presentado diferentes caminos para el desarrollo de los países, algunos de los cuales se dieron mediante la industrialización, la división internacional de trabajo, la especialización, el proceso de importación y exportación, mayor libertad económica e innovación y tecnología. Estas diferentes formas de progreso han permitido el desarrollo de ciertos países, mientras que otros han quedado rezagados.

En el marco de globalización financiera y el financiamiento del desarrollo de América Latina, se están haciendo numerosos esfuerzos para atacar cuatro componentes característicos de la región que son causa del rezago de los países: la desigualdad, la inversión, la fiscalidad y la productividad. Dentro del diseño de sus estrategias integrales actuales, los países focalizan todas las políticas económicas en dos puntos esenciales: infraestructura y matriz productiva, de manera que por medio de ellas se pueda impulsar un crecimiento más sostenido. El trade-off de inversión en infraestructura y matriz productiva implica un análisis profundo y la evaluación de alternativas que tengan importantes efectos a largo plazo, y que pueden brindar solución a estas problemáticas.

Por otra parte, el Paraguay, pese a su gran potencialidad, presenta insuficiencia de capital físico y tecnológico, lo que lo vuelve altamente vulnerable a factores exógenos como el clima y la variación de los precios internacionales de los commodities, entre otros. Además, los procesos de reforma agraria emprendidos no condujeron a los resultados esperados. Debido a esto, se sigue observando bajo acceso a créditos y un sector productivo ineficiente por la poca asistencia técnica recibida, entre otras problemáticas, lo que no contribuye con el desarrollo. 
Otro rasgo del país es que el $40 \%$ de las unidades económicas agroindustriales corresponden a microempresas y pequeñas empresas ${ }^{1}$, pero solo ocupan el $17 \%$ de la mano de obra utilizada en la industria y generan tan solo el $2 \%$ de ingresos por ventas, mientras que las medianas representan alrededor de $3.5 \%$ del total industrial, ocupan el $6.2 \%$ del total del personal industrial y poseen ingresos del $2 \%$ al igual que las pequeñas empresas. Las grandes, por su parte, representan solo el $1.5 \%$ del total industrial, pero ocupan el $25 \%$ de la mano de obra y concentran más del $53 \%$ de ingresos de toda la industria (Cresta, 2014).

La gran cantidad de microempresas y pequeñas empresas existentes en el país, aumenta la vulnerabilidad de las agroindustrias por factores externos como catástrofes naturales, caídas de precios internacionales por recesión de otros países, inestabilidad política, fallas del mercado, etc. Esto se debe principalmente a que las estas empresas tienen bajos ingresos, limitado acceso al crédito y que en la mayoría de los casos su infraestructura, logística, capacidad, servicios y tecnología es inadecuada y obsoleta.

Si a esto se le suma la falta de acceso al mercado por cuestiones culturales o incluso dificultad para establecer contactos que constituyan potenciales compradores a sus productos, la escasa o nula capacitación técnica recibida y la alta dependencia de la producción de un único rubro agrícola como es la soja, pueden apreciarse las dificultades que atraviesa el sector agroindustrial. Estas debilidades fueron identificadas en el Estudio de Desarrollo Rural Integral Dirigido al Pequeño Productor (EDRIPP) realizado posteriormente al 'Estudio sobre el desarrollo económico inclusivo de Paraguay’, EDEP (1998-200²).

El EDRIPP, mediante determinadas estrategias, y la división de los departamentos del país en subregiones según características comunes, los especializó en producciones específicas, por las ventajas comparativas que tenían, por intermedio del trabajo conjunto entre las instituciones públicas y privadas, productores, empresarios, comerciantes, universidades, etc. Posteriormente, los organizó en clústeres para producir un desarrollo sostenido e inclusivo al pequeño productor (JICA, 2011).

1 La Ley n. ${ }^{\circ}$ 4457/12 para las MIPYMES establece que en la República del Paraguay serán considerados los siguientes criterios para su clasificación: número de trabajadores ocupados y monto de facturación bruta anual. Son consideradas microempresas aquellas en las que trabajan hasta 10 personas y la facturación anual sea hasta USD 90909 (aprox.); la pequeña empresa puede ocupar hasta 30 empleados y facturar anualmente USD 454545 (aprox.) y la mediana contaría con hasta 60 trabajadores y facturaría hasta USD 1090909 (aprox.).

2 Este estudio surge a raíz de lo acontecido en la década de los noventa, relacionado con las producciones y exportaciones rezagadas, una balanza de pagos en crisis y el desempleo en aumento (JICA, 2013). 
Esa es la forma de trabajar propuesta por el EDRIPP para fortalecer la economía paraguaya, cuyo efecto directo se traduce en una menor dependencia de un rubro único y en menor exposición a factores exógenos por su valor agregado. Retomando esto, en el presente trabajo se propone la innovación e incursión en rubros no tradicionales, que tengan ventajas competitivas y excelentes perspectivas económicas, como la Stevia Rebaudiana Bertoni (en adelante, stevia), por las oportunidades y ventajas que posee.

\section{MARCO DE REFERENCIA}

La localización y su incidencia en la economía no son temas recientes en la literatura económica. Sin embargo, la globalización ha sido un proceso que ha influido notablemente en el pensamiento en los últimos años. Pese a su trascendencia, no puede soslayarse la importancia de la dimensión regional. Si bien el desarrollo teórico no siempre ha contemplado la complejidad de las regiones ni su relevancia para la economía, estas poseen sus propias relaciones en espacios específicos. Esta noción fue introducida al análisis con más fuerza en las últimas décadas y promovió el desarrollo de la estrategia de incentivar, con fondos públicos, la instalación de polos de desarrollo.

Esta idea prevaleció hasta la década de 1980 cuando, luego de consolidarse las interacciones típicas de la globalización, se retomaron las ideas de Alfred Marshall conforme a lo expresado por Corrales (2007). En la figura 1 puede apreciarse lo anteriormente expuesto.

La existencia de clústeres puede ser de mucha importancia para el desarrollo. Para analizar esta premisa, es necesario llevar a cabo algunas precisiones teóricas. El concepto de clúster fue esbozado por Marshall hacia la década de 1920. Este economista señaló que el conglomerado de compañías permite que existan economías externas, las cuales bajan los costos, contrariamente a lo que ocurre cuando la empresa actúa aisladamente. Esto obedece a que las economías externas permiten que las curvas de costo se desplacen hacia abajo (Martín Simón, 2003).

Además de las economías externas, otros autores señalan que es preciso que exista competencia y cooperación para que se desarrolle un clúster (Corrales, 2007). En lo que respecta a la competitividad, Porter (1990) explica que, en el marco del aglomerado formado por las empresas, las mismas cooperan entre sí mutuamente y como consecuencia de ello se derivan beneficios horizontales y verticales. Este autor resalta la importancia de la tecnología y señala que el clúster 
repercute en la competitividad en tres sentidos: el crecimiento de la productividad, un aumento de la innovación y un mayor estímulo para desarrollar nuevos negocios.

Figura 1. Evolución del desarrollo regional como preocupación teórica

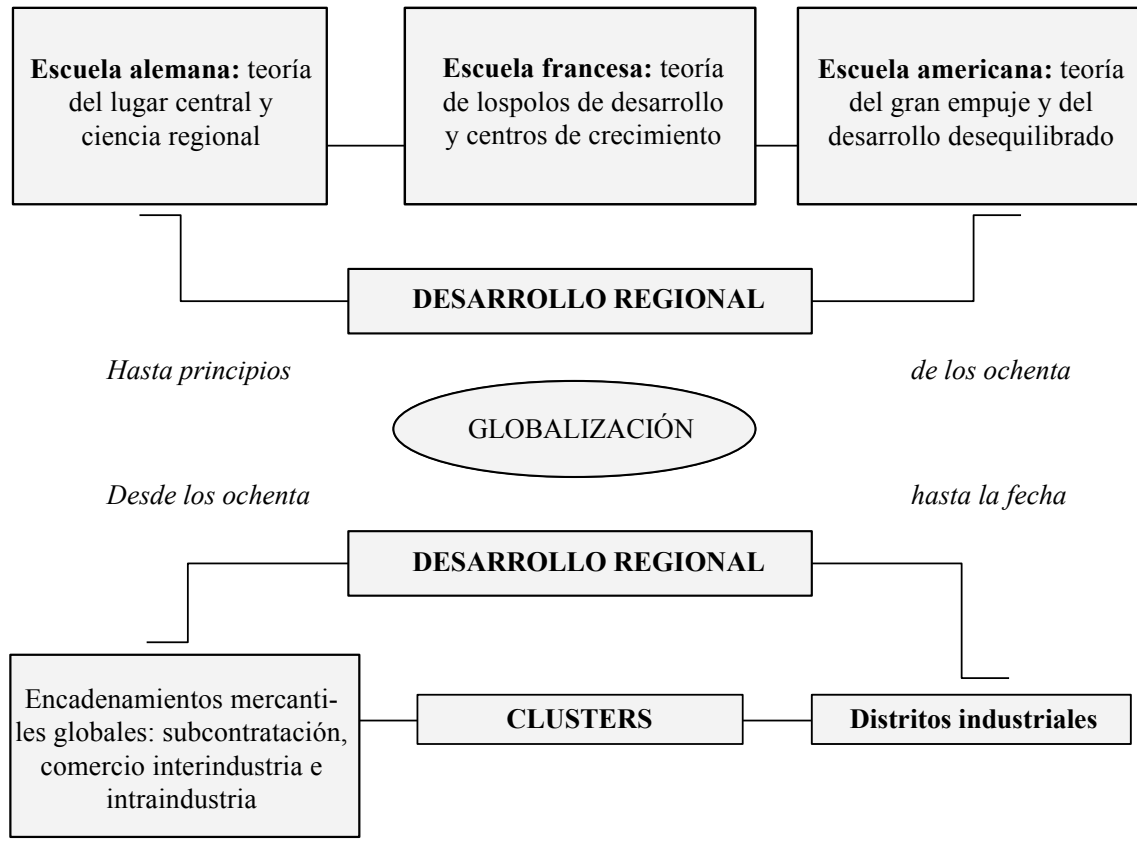

Fuente: Martín Simón (2003, p. 181).

Lo anteriormente expuesto permite apreciar que los clústeres pueden tener una notable incidencia en el desarrollo territorial, en sus diferentes dimensiones: desarrollo humano, desarrollo económico, desarrollo socioinstitucional y desarrollo sustentable. Este enfoque resulta mucho más amplio que el enfoque tradicional de desarrollo y considera al territorio como unidad de actuación y agente de transformación social. Esto se debe a que esta visión fomenta una estrategia que amalgama los esfuerzos macroeconómicos, la promoción de exportaciones y las innovaciones en los sistemas de producción local (Albuquerque, 2008).

La consideración del desarrollo territorial posibilita contemplar tres temas fundamentales para el desarrollo según Albuquerque (2008, p. 311): 
La introducción de innovaciones en los sistemas productivos locales; el diseño de los adecuados programas de formación de recursos humanos según las necesidades de cada contexto local y la referencia concreta a las características, limitaciones y oportunidades vinculadas a la valorización del medioambiente y la cultura local, esto es, la incorporación del desarrollo sustentable ambientalmente.

Tal como se había expuesto anteriormente, la inversión en infraestructura y la efectuada en la matriz productiva presentan puntos focales que impulsan el desarrollo económico (Consentino, 2015). Ambos tienen efectos importantes en la competitividad, productividad de la inversión y mejoramiento de la calidad de vida de las personas (Ruiz Díaz, 2014). No obstante, es importante mencionar que la inversión en matriz productiva posee efectos positivos en un primer estadio debido a las economías de aglomeración, para posteriormente aprovechar las economías de escala con el aumento de la producción.

Por sus características, el clúster produce movimientos en los tres sectores de una economía (primario, secundario y terciario) mediante la cooperación, asociación y actuación conjunta de los diferentes grupos o sectores público y privado, fortaleciendo así las instituciones (Piperopoulos, 2011). Teniendo en cuenta ello y que los países latinoamericanos, entre ellos Paraguay, están llamados a ser en las próximas décadas los principales oferentes de alimentos especialmente al continente asiático y al mundo entero (CEPAL, FAO \& IICA, 2009), se propone al clúster agrícola como principal estrategia.

\section{METODOLOGÍA}

En primera instancia se realizó un análisis de fortalezas, oportunidades, desafíos y amenazas (FODA) para la confirmación del rubro seleccionado. Luego se seleccionó la estrategia de financiamiento por medio de una revisión bibliográfica y la posible localización del clúster fue elegida a través de la ponderación de ciertos factores preestablecidos.

Posteriormente, se efectuaron análisis puntuales de mercado legal y financiero. Para este último caso, el flujo de caja fue proyectado siguiendo una tasa de crecimiento vegetativo para los precios del producto terminado y gastos, manteniendo el porcentaje costos/ventas constante a lo largo de los 10 años de proyección. Como referencia de tasa libre de riesgo fue utilizada la tasa de los bonos del Tesoro de Estados Unidos a treinta años. Para la estimación de la beta fue utilizada la metodología de apalancamiento y desapalancamiento de betas, desarrollada por 
los bancos de inversión para estimaciones en países emergentes donde las informaciones de mercado son limitadas. Adicionalmente, fue introducido un ajuste por riesgo país estimado por el Prof. Damodaran, el cual sigue su modelo mixto en el que el spread de riesgo soberano es una buena indicación de riesgo país, ya que indica el riesgo de default del gobierno, pero este riesgo debe ser calibrado por la volatilidad relativa del mercado accionario con respecto al de bonos. Así, el cálculo de la tasa de descuento empleada para esta valuación está basada en el siguiente modelo:

$$
\begin{gathered}
K_{e}=R f_{U S A}+\beta_{P Y} * P M_{U S A}+\text { Ajuste por Riesgo País } \\
\beta_{P Y}=\beta_{U U S A}
\end{gathered}
$$

Donde:

$K_{e}=$ costo del capital propio;

$R f_{U S A}=$ tasa libre de riesgo de EE. UU.;

$\beta_{P Y}=$ beta apalancada Paraguay;

$\beta_{U U S A}=$ beta desapalancada EE. UU.;

$P M_{U S A}=$ prima de mercado.

\section{RESULTADOS}

Se presenta en primera instancia el análisis de fortalezas, oportunidades, debilidades y amenazas (FODA) que permitía confirmar la elección del clúster de stevia como una estrategia viable. A continuación, los resultados de este estudio: Cuadro 1. FODA de la stevia 
- Cultivo autóctono con mayor calidad y pureza que los producidos por su principal competencia, China.

- Se cosecha de forma intensiva, de tres a cuatro veces al año, y no requiere de mano de obra especializada.

- Sus propiedades son conocidas mundialmente.

- Muestra alta rentabilidad y costos competitivos.
- La demanda internacional se encuentra en continuo aumento por los requerimientos de grandes multinacionales como Coca Cola, Nestlé y otras, para sustituir el azúcar.

- La proliferación de distintas patologías vinculadas al consumo de azúcar promueve su sustitución con otros productos.

- La tendencia global de consumo natural y saludable.

- $\mathrm{Su}$ consumo fue aprobado por autoridades de distintas regiones a nivel mundial.

- Bajas tasas impositivas para los bienes de capital necesarios para la producción de stevia.

- Los precios internacionales son estables.

- Se cuenta con apoyo de instituciones del Estado como la Red de Inversiones y Exportaciones (REDIEX).

- Cuenta con certificado de origen paraguayo otorgado por la Cámara Paraguaya de Stevia (CAPASTE).

\section{DEBILIDADES}

\section{AMENAZAS}

- Bajo nivel de producción y superficie sembrada.

- Dificultades de los productores ante las nuevas prácticas.

- Alto costo de producción en el primer año por la necesidad de inversión en sistema de riego.

- Problemas con la comercialización del producto.
- Potenciales competidores por los ingresos prometedores del mercado de la stevia.

- Producción de variedades mejoradas por la competencia.

- Factores ambientales y fenómenos climáticos.

- Bajo acceso a créditos, falta de asistencia técnica y capacitación.

- Instituciones altamente burocráticas.

- Coordinación entre las instituciones públicas y del sector privado involucradas en el rubro.

Fuente: elaboración propia. 
III.1. Financiación del clúster

El método de financiamiento para la formación del clúster que se ajusta a solucionar problemas del sector productivo así como a satisfacer necesidades de productos como la stevia es el Project Finance. El mismo representa una alternativa de financiamiento a la que Fight (2006: 10) define de la siguiente manera:

Una deuda sin recurso o limitada estructura de financiación con recurso en el que la mejora de la deuda, la equidad y el crédito se combinan para la construcción y explotación, o la refinanciación, de una planta en particular en una industria intensiva en capital. Los términos de la deuda se basan en los flujos de caja del proyecto.

El uso de este método se justifica por el acceso a grandes cantidades de dinero que dan la posibilidad de financiar la formación del clúster y la concreción de innumerables acuerdos y contratos que sirven de garantía y distribución de riesgos entre los participantes, formando redes. El modo de operar tanto del Project Finance como del clúster se conjuga en una alianza entre el sector privado y público.

El aprovechamiento de la formación de economías de escala mediante el clúster disminuye potenciales riesgos de iliquidez y malas previsiones de los flujos de caja estimados 3 para el Project Finance. Políticas de gobierno con visión a mediano y largo plazo basadas en el fomento del clúster evitarían la incidencia de factores políticos o cambios de gobierno en el Project Finance (Gati, 2008).

Las fuentes de financiamiento se basarían en préstamos comerciales, bonos, deuda senior, mezanine, etc., cuya garantía de devolución serían los flujos de ingresos futuros producidos por la industria (Yescombe, 2007). En la medida en que se vayan generando los ingresos por ventas de los productos finales, en los primeros años esos ingresos se constituirán para el pago de la deuda y los gastos operativos para posteriormente verse las ganancias, tanto para el sector privado como para el gobierno, considerado como un socio más.

3 Estos flujos son estimados porque las fuentes de financiamiento utilizadas mediante el Project Finance basan los servicios de la deuda en los flujos de caja futuros generados por la sociedad de vehículo especial que crea el emprendimiento. 
III.2. Localización del clúster propuesto

Para la localización del clúster de stevia con Project Finance se requerirán 300 hectáreas de tierras fiscales y ociosas, que cuenten con las condiciones agronómicas que demanda dicho cultivo, las cuales son suelos areno-arcilloso con alta capacidad de retención de humedad y precipitación media anual de $1500 \mathrm{~mm} / \mathrm{año}$ (STEVIAPAR, s. f.). Así, el departamento de Alto Paraná constituye una zona agronómica propicia para el cultivo de la stevia además de contar con 10000 hectáreas fiscales y ociosas. Para el año 2014, dicho departamento es considerado como el segundo productor de stevia siguiendo a San Pedro, con el $10 \%$ del total producido a nivel país (MAG, 2014). Si bien su producción es baja, dispone de infraestructura y servicios que facilitan la producción y comercialización. En lo que a transporte respecta, cuenta con una ruta pavimentada, que es ramal de la supercarretera número 7 , conector hacia los principales mercados tanto internos como externos, además de tener acceso a transportes aéreos y fluviales (JICA, 2011).

Dentro de este departamento, se localizó que el distrito de Ytakyry se encuentra cercano a estos puntos de transporte y cuenta con una alta concentración de tierras fiscales. Cabe mencionar que este lugar presenta antecedentes de problemas y disputas por la falta de derechos de propiedad definidos y una cultura reacia al cambio, baja apuesta e interés por la innovación en rubros con potencialidades y desconocimiento de los grandes beneficios del mercado de stevia (CADEP, 2011), por lo que este tipo de emprendimiento podría colaborar a darle una nueva perspectiva a la zona en la que se establece.

\section{III.3. Análisis puntuales de mercado, legal y financiero}

Estimación de la demanda de stevia

La stevia se presenta como una excelente alternativa para la disminución de enfermedades como la diabetes y la obesidad, e inclusive para problemas de presión arterial y sistema renal, entre otros (Steviamarket, 2015). En efecto, China lidera el grupo de los países top ten con diabetes, siguiéndole India, EE. UU., Brasil, Rusia, México e Indonesia. La prevalencia global de esta enfermedad en el 2015 era de $8.80 \%$ y en el 2040 se pronostica el $10.40 \%$ (IDF, 2015). Por su parte, en Paraguay, el número de casos en unos pocos años ha pasado del $6.5 \%$ de la población (2004) al 9.7 \% (2014) y ha ido aumentando a raíz del sedentarismo, una mala alimentación con alto contenido graso y azúcares (MSP, s. f). 
Por otra parte, la obesidad y el sobrepeso aumentaron en un $50 \%$ y $19 \%$ respectivamente en el período 2005-2015, constituyéndose EE. UU., China, India, Brasil y México en los países con mayor población aquejada con estos padecimientos (WHO, 2015). En Paraguay, según el Programa Nacional de Diabetes (2014), el 57.6 \% de la población debe enfrentar estos problemas, es decir, 6 de cada 10 paraguayos.

Existen diferentes productos de edulcorantes acalóricos, tanto artificiales como naturales, para combatir estas enfermedades. No obstante, la ventaja de la stevia frente a los edulcorantes artificiales como el aspartame, el ciclamato, la sucralosa, etc., radica en los efectos que tienen estos en el organismo.

Por otra parte, al considerar los edulcorantes naturales y comparar la stevia con otros edulcorantes de la misma clasificación pero de diferente subgrupo tales como los monosacáridos y oligosacáridos, se evidencia que dosis masivas de consumo de los componentes de dicho grupo, como son la fructosa, sacarosa, jarabe de maíz y de agave, constituyen la principal causa de obesidad y aumentan el riesgo de contraer diabetes (Mercado, 2015).

A raíz de la prevalencia de enfermedades expresada anteriormente y del conocimiento mundial de los atributos de esta planta, se proyecta un aumento de la demanda de productos naturales como la stevia. Se pronostica que su consumo será de 8506.9 toneladas hacia el término del 2020 teniendo un crecimiento anual de 7-8 \% durante el período 2016-2020 (Steviamarket, 2015).

Las circunstancias detalladas, sumadas a la escasa oferta, hacen necesario que en los países productores como Paraguay se creen economías de escala que puedan atender al menos parte del mercado. Para la creación de estas economías, la formación de clústeres representa la más eficiente alternativa de organización y es la manera de operar que mejor se adapta a dicho producto agrícola, atendiendo el trabajo conjunto y coordinado que requiere, así como también las características y problemas que presenta el sector agrícola del país.

El mercado potencial consumidor se basa en variables geográficas y conductuales. Las variables geográficas se centran en el mercado meta externo e interno. En el mercado meta externo se tienen en cuenta a los países con los que ya se mantuvieron relaciones comerciales tanto en materia de las hojas secas de stevia como el steviosido ${ }^{4}$. Así, los principales mercados para las hojas secas de stevia

4 El steviosido es un extracto en polvo de la stevia mientras que la stevia es la hoja seca. 
procedentes de Paraguay son China, que lleva casi todo el mercado, con el $90 \%$, mientras que el resto se distribuye entre Francia con el $5 \%$, México $1.4 \%$, Argentina y EE. UU. con el $0.7 \%$ (BCP, 2015). En cuanto al mercado de steviosido, los principales países son EE. UU. y Brasil correspondiéndole el $36 \%$ y $35 \%$, respectivamente.

Según la American Diabetes Association (2014), esta enfermedad es considerada como una de las principales causas de muerte en EE. UU., por lo que si se considera aquella población con probabilidades de adquirirla para los próximos años, se podría generar una demanda aproximada del mercado potencial. Así, se tiene una población base de $318.9^{5}$ millones de habitantes para el 2014, un crecimiento anual de $0.8 \%^{6}$, prevalencia del $14 \%{ }^{7}$ y cantidad máxima requerida al día de steviosido de 1000 miligramos $^{8}$. A partir de estos datos se estimó la participación del steviosido de la potencial industria. En los siguientes gráficos, se pueden observar la fracción de la población con diabetes a partir de la población total y la participación del steviosido a partir del consumo potencial.

Se estima que la población con diabetes pasará de 45.36 millones de habitantes en el 2016 al 57.61 habitantes en el 2046. La demanda estimada para el 2016 sería de 16.557 toneladas, por lo que se toma $0.67 \% 9$ de participación en aquel mercado. Dicha proporción es muy conservadora debido a la capacidad de la industria para atender la gran demanda y a que la competencia del azúcar es aún fuerte. Lo dicho se puede observar en el gráfico 2. No se puede percibir la participación del steviosido en el mercado por el amplio margen entre demanda y oferta.

\footnotetext{
Dato extraído del World Bank.

Dato extraído del World Bank.

Extraído de la Federación Internacional de la Diabetes, 2015.

Extraído de la FAO.

9 Esta cifra ajusta a la cantidad total de producción estimada por año dado que la misma es proporción de la demanda estimada de steviosido de la población con diabetes y el resultado de ello se aproxima a la cantidad producida anualmente.
} 
Gráfico 1. Población total y con diabetes estimadas

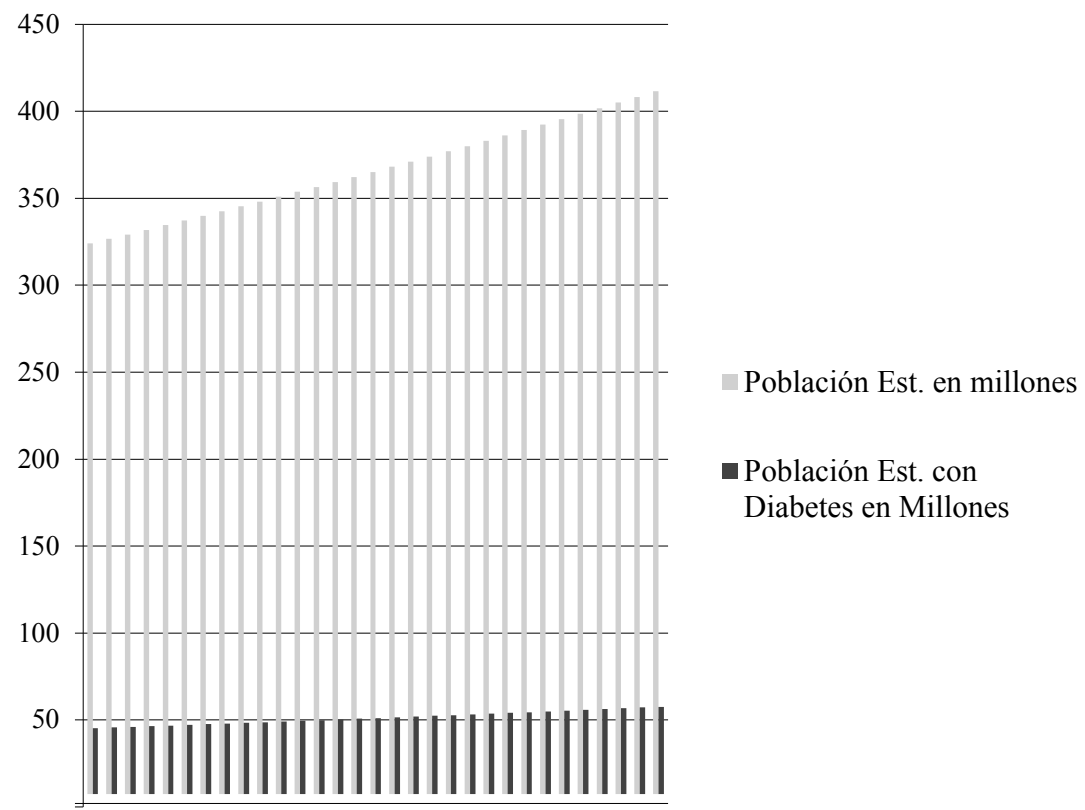

Fuente: elaboración propia sobre la base de datos de la American Diabetes Association (2014).

Gráfico 2. Participación del steviosido a partir del consumo

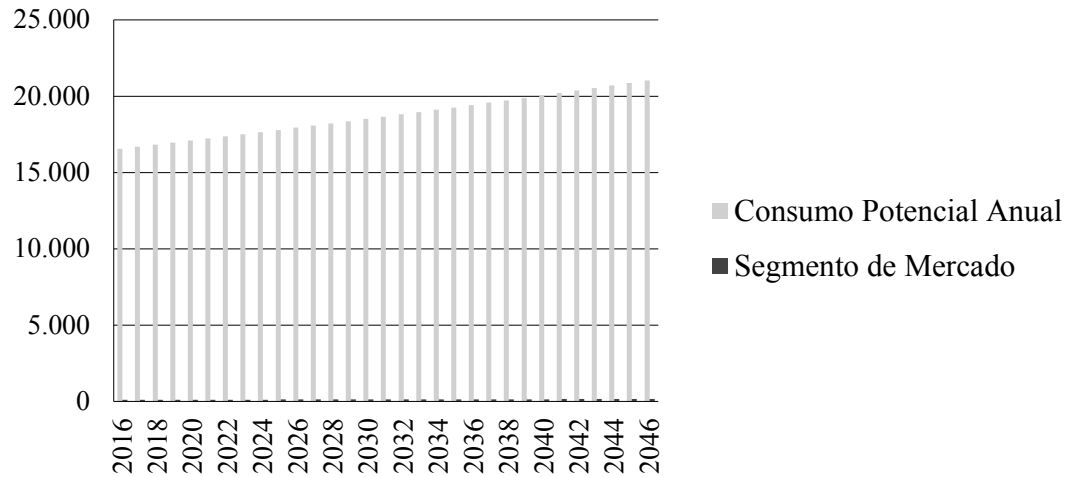

Fuente: elaboración propia. 
En cuanto a la determinación del mercado meta interno, se busca captar a los consumidores del azúcar ${ }^{10}$ de tal forma que el steviosido constituya un sustituto saludable y con menor aporte en calorías. Los principales departamentos focos serán los que tengan mayor densidad poblacional, tal es el caso de Central con el $34 \%$ de la población total, Alto Paraná con el $12 \%$, Itapúa con el $8.1 \%$ y Asunción con el 7,5\% (DGEEC, 2013).

Por consiguiente, para definir la participación en el mercado interno, se calculó el consumo aparente histórico del azúcar en el país, para lo que se recurrió a la siguiente igualdad: consumo aparente de azúcar $=$ producción total + importación - exportación. Para dicha ecuación no se tuvieron en cuenta datos no registrados, como los del contrabando, que también inciden en el consumo del rubro (gráfico 3).

Gráfico 3. Producción, importación, exportación y consumo históricos del azúcar

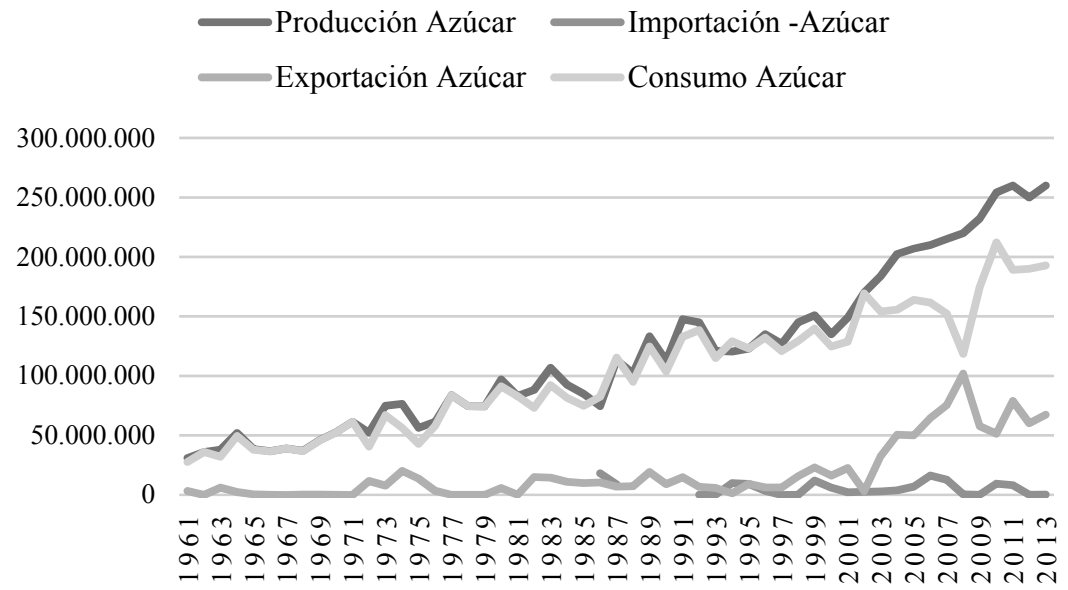

Fuente: elaboración propia sobre la base de la FAO.

Se puede observar la gran diferencia entre la producción y los volúmenes de las transacciones externas. Las exportaciones a partir del 2003 han tenido un progresivo aumento mientras que las importaciones han permanecido muy bajas en comparación con el total producido. El consumo acompaña la producción por

10 Con base en el histórico del consumo de azúcar se proyecta el consumo aparente del steviosido. 
lo que se puede ver el sólido mercado del azúcar en el país. Al observar el comportamiento del consumo en aquel período, se percibe que sigue una tendencia definida y positiva.

Teniendo en cuenta que no existe estacionalidad, se tiene la recta de mejor ajuste 2. $\mathrm{Y}=3097957 \mathrm{X}+18591238$ (gráfico 4). El consumo aparente de azúcar viene explicado en un $92 \%$ por la variable independiente 'años'. Podría decirse también que por cada año que transcurre, el consumo de azúcar aumenta 3097957 kilogramos, es decir 3098 toneladas.

Tomando como base aquella ecuación, se proyecta el consumo aparente para el período 2016-2046, tomándose un segmento del mercado por cada año que transcurre. Dicha proporción, al igual que el mercado externo, es conservadora por los motivos ya mencionados. En el gráfico 5, se muestra la proyección de la participación en el mercado a partir de la demanda interna aparente pronosticada.

Gráfico 4. Comportamiento del consumo aparente del azúcar11



Fuente: elaboración propia sobre la base de la FAO.

11 El consumo aparente de azúcar permitió obtener el comportamiento de esta variable y considerando que la utilización del steviosido podría ser un sustituto de la misma, se obtendría entonces su potencial consumo. 
Gráfico 5. Participación del steviosido en el mercado interno

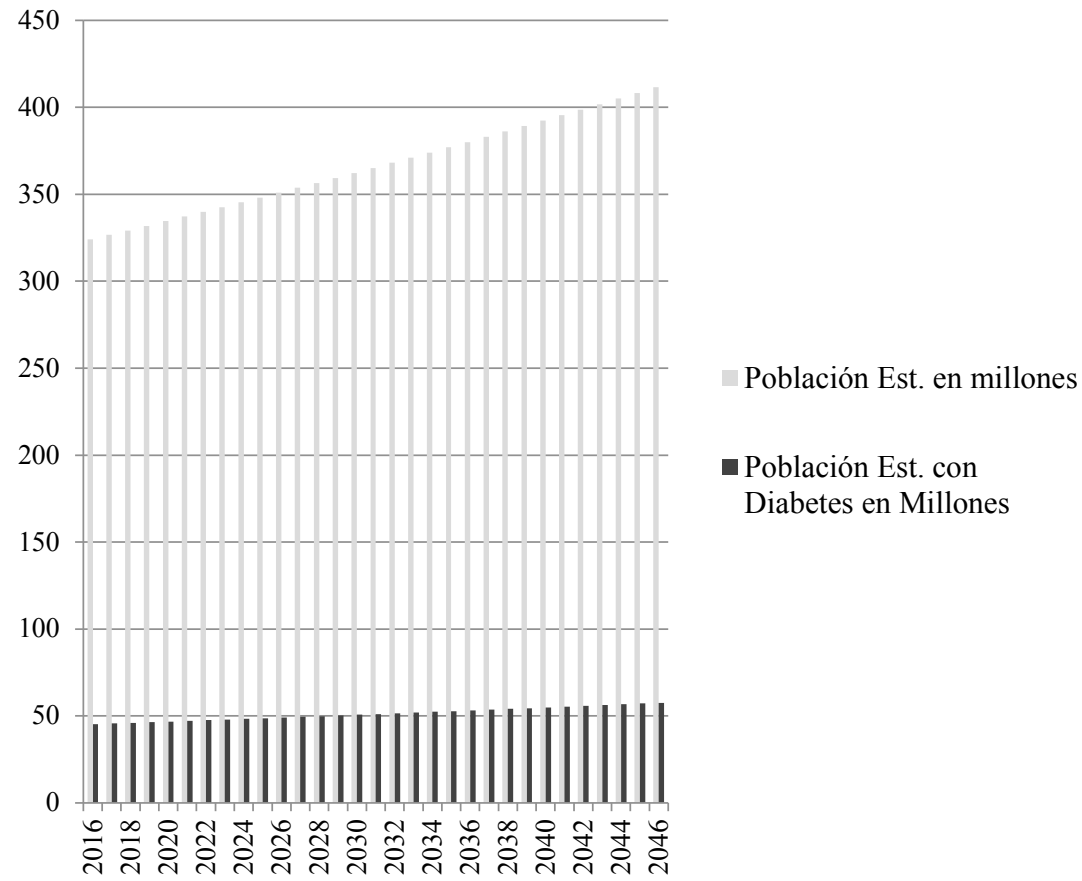

Fuente: elaboración propia sobre la base de la FAO.

El steviosido participa con un $0.038 \% 12$ del consumo en el primer año, equivalente a 73 toneladas, aumentando esta cantidad para el 2046 a 103 toneladas. Su participación, al igual que el mercado externo, es insignificante por la incapacidad de abastecer el mercado.

En cuanto al aspecto legal de la utilización del Project Finance en el clúster de stevia se hace necesario resaltar ciertos artículos de la ley n. ${ }^{\circ}$ 5102/13 "De la Promoción de la inversión en infraestructura pública y ampliación y mejoramiento de los bienes y servicios a cargo del Estado" para ver las posibles ampliaciones y analizar cómo podrían favorecer al tipo de emprendimiento propuesto.

12 Está proporción se complementa con el resultado de la cantidad consumida externamente que trata de ajustarse a la oferta productiva. 
Cuadro 2. Análisis de la Ley 5102/13

Normativas actuales

El artículo 3 en su párrafo II menciona el compromiso establecido en contrato del participante privado, que incluye como mínimo el financiamiento total o parcial de las inversiones.

El artículo 12 hace referencia a los pagos ofrecidos por el sector privado a la administración contratante del Estado por motivo de superávits en los ingresos o lo estipulado en el contrato de APP.

Los artículos 26 y 31 hacen mención a la constitución de garantías del sector privado mediante avales fianzas, cartas de crédito.

Artículo 38. El sector privado podrá financiar el desarrollo de proyectos mediante instrumentos y modalidades financieras utilizadas en el mercado financiero nacional o internacional.

\section{Normativas ampliadas y/o analizadas}

Este artículo debería hacer mención al Project

Finance como opción de financiamiento del participante privado en el caso de no contar con el financiamiento total o parcial.

Este artículo, al considerar "Lo estipulado en el contrato de APP”, da la posibilidad de crear la figura del Estado* como un accionista más de la sociedad y poder recibir un porcentaje de las utilidades en concepto de ello.

En este se debe tener en cuenta que bajo la modalidad del Project Finance no existen garantías reales sino que se basan en los flujos de caja esperados y de los activos de la sociedad creada.

En este artículo, se hace mención de forma implícita al Project Finance, instrumento utilizado a nivel nacional e internacionalmente.

* Esta figura se crea bajo la modalidad del Project Finance en clúster por el trabajo conjunto de los sectores público y privado. Fuente: elaboración propia.

Análisis de factibilidad

El siguiente análisis de factibilidad de la instalación de la industria de stevia considera una valuación hecha "al infinito" en forma de perpetuidad creciente para recoger el horizonte infinito de la valuación. Esta fue hecha tanto a nivel de la empresa (antes del financiamiento) como de los potenciales promotores de la inversión. Atendiendo el objetivo de esta valuación, el modelo seleccionado es el de descuentos de flujos (DCF), el cual utiliza flujos de caja libres tanto para la empresa como para los accionistas.

Este modelo se basa en que el valor de un activo deriva del potencial de generación de caja futura que el mismo posee, el cual es descontado a una tasa por 
el riesgo asociado a dichos flujos. Por tanto, el valor depende de la magnitud de los flujos de caja, el tiempo, la incertidumbre de esos flujos, la tasa de interés de mercado y las perspectivas de crecimiento. Se considera como base del análisis los flujos de caja libres tanto para la empresa (Free Cash to the Firm, FCFF) como para el accionista (Free Cash to the Equity, FCFE), que representan los fondos generados para la empresa y el accionista de la empresa, respectivamente (Buhk, 2016).

Para el flujo de caja para las empresas o FCFF se utilizó la siguiente fórmula: ganancias de la operación - inversiones que salen del balance. Para el FCFE se parte de ganancia neta - inversiones - los pagos de la deuda. Luego dichos flujos se descuentan a sus correspondientes tasas. El Weighted Average Cost of Capital (WACC) o costo medio ponderado de capital es utilizado para el FCFF y el Cost Equity (Ke) o costo de capital propio para el FCFE (Roca, 2012).

Algunas limitantes del análisis son la falta de un mercado activo de formación de precios a nivel país, lo que dificulta toda estimación de riesgo necesaria en cualquier valuación. Además, es preciso señalar que la información utilizada para realizar este trabajo se basa íntegramente en datos proveídos por una industria de stevia, de los que se asume la veracidad, ante la imposibilidad de encontrarlos en otra fuente.

Los supuestos en los cuales se basó la valuación fueron:

1. Depreciación lineal en 10 años de los siguientes activos edificios, maquinarias, instalaciones, mobiliarios y oficina, etc. Aquí se incluye la tecnología know how, a la que se considera una amortización, que junto con la depreciación constituyen los gastos no desembolsables.

2. La estructura de costos (materia prima, insumos generales, tratamiento de efluentes, agua, caldera y control de calidad) fue proyectada a un porcentaje constante ${ }^{13}$ de los ingresos, a partir de los costos de producción y las toneladas vendidas de la industria de stevia.

3. El precio por tonelada fue proyectado a tasas de crecimiento del $3 \%$ considerando la inflación meta.

13 Se considera un porcentaje constante debido a que el mercado de la industria de la stevia es estable y dicho ratio se mantiene, esto se constata con la entrevista realizada a la agroindustria de stevia que opera en el país. 
4. Para los gastos se consideró la información proveída por la industria de stevia, los cuales presentaban recargos adicionales por cada 50 toneladas más que se producían. A partir de ello, se calcularon los gastos sobre la base de la producción proyectada. Se tuvieron en cuenta los recargos, en caso de que los hubiere, aumentando anualmente el $3 \%$ correspondiente a la inflación. Los gastos de mantenimiento de maquinarias, equipos y vehículos se incluyeron en esta cuenta de gastos.

5. Fue asumido un rendimiento de 3200 kilogramos por hectárea, por los equipos de riego e industriales y el proceso productivo inocuo (Candia, 2016, comunicación personal).

6. Se considera un gran nivel de endeudamiento producto de la insuficiencia de capital de los promotores de la inversión para con este tipo de inversión.

7. Se supone la utilización de la metodología de financiamiento del Project Finance por lo que no existen garantías reales por parte de los promotores y por ello los flujos de caja futuros constituyen el principal centro de interés de los inversionistas institucionales (proveedores de financiamiento).

8. La estructura de financiamiento se basó en aporte propio de $15 \%$ y deuda $85 \%$, este último distribuido en fondos de instituciones financieras, BID-FOMIN, IFC, FIDA, CAF y bonos.

9. El costo de la deuda es resultado del promedio de las tasas bursátiles ${ }^{14} \mathrm{y}$ bancarias. Se consideró el plazo de 10 años para el pago total de la deuda, utilizándose el sistema francés.

10. Se considera el histórico del consumo de azúcar para proyectar el consumo aparente del steviosido por su carácter de sustituidor.

\section{II.4. Estimaciones realizadas}

Tal como se había descripto en el apartado correspondiente a la metodología, se estimó la beta mediante la metodología de apalancamiento y desapalancamiento de betas y se incorporó un ajuste por riesgo país estimado por el Prof.

14 Se incluyen deudas bursátiles debido a la metodología utilizada de Project Finance, la cual se vale de diferentes fuentes de financiamiento, entre ellas las bursátiles. 
Damodaran ${ }^{15}$, el cual sigue su modelo mixto. Este explica que el spread de riesgo soberano es una buena indicación de riesgo país. Los resultados obtenidos fueron las siguientes tasas de descuento: WACC del $10 \%$ y Ke del $34 \%$.

Resultado de la valuación

\section{Proyecto antes de considerar el endeudamiento}

Según las estimaciones realizadas, el valor del proyecto a nivel empresa resultó en USD 52 millones (VAN del proyecto) y una tasa interna de retorno (TIR) del $38 \%$. El período de recupero descontado de la inversión es de 4 años. Los detalles de la estimación se encuentran resumidos en el gráfico 6 .

Los flujos de caja libres para la empresa ${ }^{16}$ que se encuentran disponibles, ya sea para cualquier inversión que desee realizar o para la focalización de las miradas de los proveedores de créditos por la liquidez y solvencia que posean (para sus posteriores pagos), van desde $\$ 4 \mathrm{MM}$ en el año 1 hasta los flujos generados hasta el infinito ${ }^{17}$ \$ $93 \mathrm{MM}$. De esta manera, se observa que la industria establecida para convertirse en economía de escala tiene resultados sumamente alentadores para los agentes económicos.

15 Se recomienda la lectura del material existente en este link para obtener más detalles de la metodología empleada por Damodaran: http://pages.stern.nyu.edu/ adamodar/; http://www.treasury.gov/ resource-center/data-chart-center/interest-rates/Pages/TextView.aspx?data=yield

16 Estos flujos de caja son los disponibles para la empresa después de descontar todos los costos de producción, gastos, depreciaciones e impuestos sin considerar intereses porque no se cuentan las deudas.

17 El valor terminal es el valor de todos los flujos de caja posteriores (hasta el infinito) a un período proyectado del proyecto (generalmente 10 años), que se presenta en un único monto. Este se calcula por la siguiente formula, $F c n *(1+g) / r$ - $g$. Los mismos representan, $F c n=$ Flujo de Caja en el último año estimado; $r$ = tasa de descuento; $\mathrm{g}=$ tasa de crecimiento a largo plazo o tasa de perpetuidad. 
Gráfico 6. Resultado antes de considerar el endeudamiento FCFF

VAN US\$ $52 \mathrm{MM}$

TIR 38\%

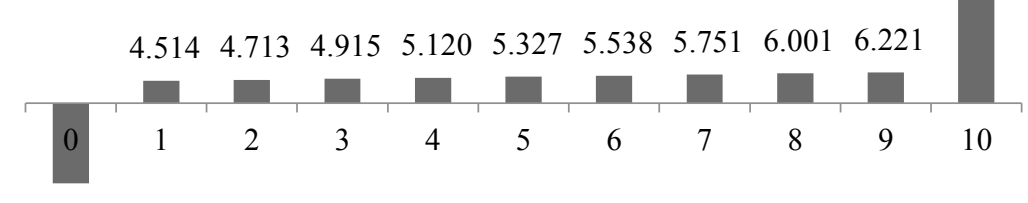

$(16.150)$

\section{Tasa de Descuento \\ WACC 10\%}

Fuente: elaboración propia sobre la base a datos proveídos por industria.

\section{Proyecto después de considerar el endeudamiento}

Según las estimaciones realizadas, el valor del proyecto a nivel empresa resultó en USD 6 millones (VAN del proyecto) y una tasa interna de retorno (TIR) del $118 \%$. El período de recupero descontado de la inversión es de un año. Los detalles de la estimación se encuentran resumidos en el gráfico 7.

Téngase en cuenta el alto grado de apalancamiento que el Project Finance otorga a los promotores de la inversión; aporte propio $15 \%$ y deuda $85 \%$. Por lo tanto, el período de recupero de lo invertido para los accionistas sería de un año por su bajo aporte. También se puede observar que los accionistas después de pagar las deudas y los intereses tienen disponibles para ellos desde \$2 MM en el año 1 a $\$ 15$ MM generados hasta el horizonte temporal infinito. A través de estos indicadores financieros del VAN y TIR de los FCFF y los FCFE, se puede observar lo rentable y beneficiosa que es la industria para los que promueven la inversión, los que invierten y la sociedad en general. Esto se confirma aún más en el análisis de los escenarios. 
Gráfico 7. Resultado después del financiamiento

VAN US\$ 6MM

TIR 118\%

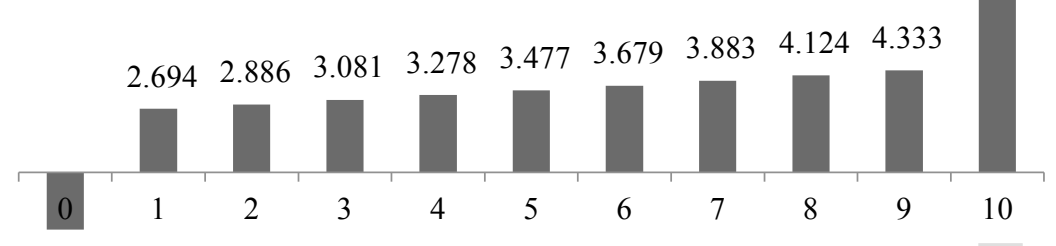

$(2.422)$

\section{Tasa de Descuento}

Ke 34\%

Fuente: elaboración propia.

\section{II.5. Análisis de escenarios}

Fueron consideradas las variaciones de dos variables: los costos con relación a las ventas y el riesgo país, que incide en las tasas de descuentos, tanto el WACC como el Ke. Este análisis muestra que aún bajo el escenario pesimista, donde los costos sean $60 \%$ de las ventas ( $10 \%$ más de la estimación base) y un riesgo país $1.5 \%$ más elevado, el proyecto sigue siendo aceptable (tabla 1 ).

Con este resultado se confirma la sostenibilidad en el tiempo de esta industria y su formación en clúster de stevia (economía de escala) al utilizar la metodología del Project Finance. A partir de ello, se puede observar el impacto de su implementación en toda la economía. Si se considera la medición del PIB desde la óptica del flujo de los ingresos (salarios, intereses, rentas) o costes generados de producir los productos, es posible apreciar el efecto de la implementación de la propuesta. 
Tabla 1. Escenarios

\begin{tabular}{|c|c|c|}
\hline & FCFF & FCFE \\
\hline \multicolumn{3}{|l|}{ Escenario Base } \\
\hline VAN & 51.735 & 6.316 \\
\hline TIR & $38 \%$ & $118 \%$ \\
\hline Periodo de Recupero & 4 & 1 \\
\hline Indice de Rentabilidad & 1,62 & 3,93 \\
\hline \multicolumn{3}{|l|}{ Escenario Optimista } \\
\hline VAN & 80.649 & 9.434 \\
\hline TIR & $45 \%$ & $148 \%$ \\
\hline Periodo de Recupero & 4 & 1 \\
\hline Indice de Rentabilidad & 5,99 & 5,44 \\
\hline \multicolumn{3}{|l|}{ Escenario Pesimista } \\
\hline VAN & 36.260 & 3.522 \\
\hline TIR & $34 \%$ & $81 \%$ \\
\hline Periodo de Recupero & 6 & 3 \\
\hline Indice de Rentabilidad & 3,25 & 1,71 \\
\hline
\end{tabular}

Fuente: elaboración propia.

En efecto, en promedio se estarán creando 5256 empleos más anualmente en todo el departamento de Alto Paraná, resultado de implantar e iniciar la formación del clúster en Ytakyry. Dicho dato se sustenta en los requerimientos de mano de obra por hectárea, según investigaciones sobre los costos de producción de la Dirección de Extensión Agraria del Ministerio de Agricultura y Ganadería en el 2015. Esta demanda varía dependiendo del año de producción en que se encuentre la stevia, variando de 125 personas en el primer año a 66 en el cuarto, por hectárea. En cuanto a los empleos industriales generados en el departamento, se estima un total de 70 personas más en promedio anualmente. Finalmente, es interesante mencionar que los ingresos que reciben los agricultores e industriales aumentarían, pasando de un total pagado anualmente de 65.601 USD (promedio anual de ingreso para los dos sectores 77.266 USD y 245 USD respectivamente) en el 2016 a 142.759 USD en el 2046. 
Otro aspecto a considerar se relaciona con la asignación de los derechos de propiedad. Si se observa la historia, se comprueba que son mucho menores los problemas sociales que surgen cuando los derechos de propiedad están definidos. Por tanto, la concesión de las tierras del gobierno al sector privado, así como el fiel cumplimiento de los innumerables contratos que demanda la utilización del Project Finance, acompañado de las políticas de transparencia aplicadas actualmente, contribuirán a mejorar la seguridad jurídica del país permitiendo proyectar una mejor imagen a los demás países.

De esta manera, la mayor transparencia de las instituciones y el aumento de la seguridad jurídica incidirán en el índice de competitividad global que actualmente ubica a Paraguay en la posición 118 de un total de 140 países analizados en el ranking. Para que el posicionamiento en dicho índice sea cada vez mayor y que cada país pueda escalar y ocupar mejores posiciones se tienen en cuenta 12 variables, las cuales inciden en el resultado final. La incidencia más notable puede apreciarse en variables como instituciones, infraestructura, educación superior y formación, eficiencia del mercado de bienes, desarrollo del mercado financiero, preparación tecnológica, tamaño del mercado, sofisticación en materia de negocios, innovación y eficiencia del mercado laboral (WEF, 2015).

En efecto, mediante este modelo de clúster con Project Finance se genera una mayor formalidad e innovación en las empresas, lo que redunda en mayor competitividad. Cabe resaltar que la competitividad global es uno de los factores que atrae inversiones y que las inversiones traen consigo mayor desarrollo económico (Servín, 2011).

Contar con medios de financiamiento como el Project Finance otorga la oportunidad de invertir en mejores infraestructuras, tecnologías y capital humano de tal manera que la informalidad desaparezca. Esta informalidad se observa en el escaso acceso a créditos y la ausencia de trabajadores bien calificados (Banco Mundial, 2010).

Por otra parte, la innovación de las empresas es vital para el desarrollo económico de un país (Banco Mundial, 2010). Así como menciona De la Torre (2016), la permanencia de las empresas paraguayas es de solo 30 años aproximadamente. Esta corta existencia puede deberse a la falta de innovación constante por parte de las empresas para mantenerse en el tiempo. Es por ello que participar de cadenas productivas, lograr una fuerte integración de las etapas productivas, aprovechar los recursos naturales con los que se cuenta, así como la constante innovación de las empresas, es de vital importancia. 


\section{CONCLUSIÓN}

Innovación, mentes creativas, sectores estratégicos, aprovechamiento de oportunidades y trabajo conjunto son los puntos principales tratados en esta investigación.

Hoy, cada vez más, el proceso de globalización exige a países de América Latina como Paraguay llevar a cabo estrategias, implementar nuevos métodos, desarrollar innovaciones a partir de la visualización de experiencias internacionales, identificar debilidades del sistema en el que se configuran y oportunidades, ventajas, etcétera.

De esta forma, se considera la combinación de clúster con Project Finance como potencial estrategia para la generación de beneficios tanto económicos como sociales desde 2016 a 2046. Por medio de la investigación realizada, se pudo confirmar que la identificación correcta del sector económico, y en este caso el rubro productivo (la stevia), es vital para que aquel modelo clúster-Project Finance funcione.

En efecto, esta forma de trabajo supone un alto grado de apalancamiento de deuda por parte del sector privado, en donde la característica principal es que la devolución de la deuda y los intereses no cuentan con garantías reales, sino que se basan en los flujos de caja futuros generados por la actividad principal. Bajo esta forma de trabajar se hace imprescindible la cuantificación de los potenciales flujos de caja generados por la industria. Los resultados confirman las perspectivas favorables de inversión en la industria de stevia y de convertirla en una economía de escala por la rentabilidad que presenta en el tiempo, y por las amplias oportunidades de mercado, no solo por una baja oferta sino por la gran demanda actual y potencial que posee.

Clúster con Project Finance es la combinación propuesta, la cual engloba y se ajusta a las ideas expuestas. En efecto, los beneficios económicos y sociales generados primeramente a nivel micro (nivel empresa, accionistas, inversionistas, trabajadores y productores del clúster) y trasladados a nivel macro (efecto derrame sobre el departamento y el país en general) crean un mayor desarrollo económico, haciendo menos dependiente la economía de los commodities y protegiéndola de factores exógenos por el valor agregado que incorpora la agroindustria. Así, la integración del Project Finance con clúster en el departamento de Alto Paraná propiciaría un mayor desarrollo con la inclusión de sectores tradicionalmente excluidos.

Otras cuestiones que se solucionan a nivel micro, y que tienen su impacto a nivel macro, son los problemas de la tierra generados por la falta de definición y asignación de los derechos de propiedad de tierras fiscales. Al otorgar en conce- 
sión dichas tierras de Ytakyry, que no son propiedad de nadie y que son motivos de disturbio, se resuelve dicho problema social. Esto impacta a nivel macro en la medida que asegura que el país proyecte una mejor imagen en el exterior, en cuanto a seguridad jurídica se refiere. Ello, acompañado de políticas de transparencia del gobierno, ayuda a fortalecer las instituciones y su credibilidad. Problemas como la informalidad también se logran atacar debido a que las empresas tienen oportunidad de acceder a créditos mediante la metodología del Project Finance.

Además, el escaso interés por la producción de stevia de los agricultores debido a la exigencia de cuidados culturales e intensivos que requiere la planta y la falta de apoyo del gobierno mediante asistencia técnica, hace necesario que se demuestre un fuerte respaldo al rubro, para suscitar un mayor compromiso de todos los actores implicados.

\section{REFERENCIAS BIBLIOGRÁFICAS}

Banco Central del Paraguay. (2015). Informe de Comercio Exterior. Recuperado de https://www.bcp.gov.py/informe-de-comercio-exterior-mensual-i466

Banco Mundial (2010). Desarrollo de Indicadores. Recuperado de https://datos. bancomundial.org/indicador

Buhk, S. (2016). Modelo de Valuación de Flujos de Caja.

CADEP (2011). Inserción de Paraguay en el Mundo. Diagnóstico y Lineamientos. Mesa del Sector Productivo.

CEPAL, FAO \& IICA (2009). Perspectiva de la Agricultura de América Latina. Recuperado de https://www.cepal.org/es/publicaciones/1376-perspectivasla-agricultura-desarrollo-rural-americas-mirada-america-latina

Consentino, A. (2015). Conferencia Globalización Financiera y el Financiamiento del Desarrollo. Centro de Convenciones de la UNA.

Cresta, J., Velaztiqui, J., Garay, P. \& García, A. (2014). Sector Agroindustial de Paraguay. Banco Interamericano de Desarrollo. Recuperado de https:// publications.iadb.org/handle/11319/6746? scope $=123456789 / 11 \&$ thum bnail $=$ false \&order $=$ desc \&rpp $=5 \&$ sort_by $=$ score \&page $=1 \&$ query $=$ mar ine + energy + in + chile $\&$ group_by $=$ none $\&$ etal $=0 \&$ filtertype_ $0=$ country en\&filter_0 $=$ Paraguay\&filter_relational_operator_ $0=$ equals

De la Torre, A. (2016). Conferencia 'La gran desaceleración Latinoamericana: Desafíos Macroeconómicos y en el mercado de trabajo'. Sala de Convenciones del Banco Central del Paraguay.

FAO (s. f.). Base de datos de Producción y Comercio de Paraguay. Recuperado a partir de http://www.fao.org/faostat/en/\#data/QD 
Fight, A. (2005). Introduction to Project Finance. Reino Unido: Elsevier.

Gatti, S. (2008). Project Finance in Theory and Practice: Designing, Structuring, and Financing Private and Public Projects. Academic Press Advanced Finance Series. London: Academic Press.

International Diabetes Federation (2015). IDF Diabetes Atlas (Seventh). Bruselas: Karakas Print.

Informe del Programa Nacional de Diabetes (2014). Ministerio de Salud Pública y Bienestar Social. Recuperado de http://portal.mspbs.gov.py/dvent/ programa-nacional-diabetes/

Irimia, A. \& Oliver, M. (s. f.). Estudio sobre la Financiación Privada de Infraestructuras (Escuela de Economía de Andalucía). Sevilla: Elsevier.

JICA (2011). Estudio para el Desarrollo Rural Integral Dirigido al Pequeño Productor (EDRIP). Paraguay. Recuperado de http://open_jicareport.jica. go.jp/pdf/12041778_01.pdf

JICA (2013). Estudio sobre el Desarrollo Inclusivo en el Paraguay. Experiencias de una Cooperación Internacional. Naciones Unidas. Recuperado de https:// www.jica.go.jp/paraguay/espanol/office/others/c8h0vm0000ad5gke-att/ info_09.pdf

Mercado, M. E. (2015). Edulcorantes y sus efectos en la Salud. VII Simposio Internacional del Ka'a He'e-Stevia. Recuperado de https://www.researchgate. net/publication/312899595_Ka'a_he'e_Stevia_rebaudiana_Bertoni_Bertoni La_dulce_planta_de_Paraguay_para_el_mundo_alternativa_para_la_ diversificacion_de_la_finca

Ministerio de Salud Pública y Bienestar Social (s. f.). Datos sobre Sobrepeso, Obesidad y Diabetes en Paraguay. Recuperado de https://www.paho.org/ par/index.php?option=com_content\&view $=$ article \&id=1010:dia-mundialdiabetes\&Itemid $=258$

Piperopoulos, P. G. (2011). Entrepreneurship, Innovation and Business Clusters. Farnham: Gower. Recuperado de: https://www.researchgate.net/ publication/268806001_Entrepreneurship_Innovation_and_Business_ Clusters

Ruiz Díaz, F. (2014). Las alianzas público-privadas. ¿Interés general versus interés particular? Recuperado de http://www.cadep.org.py/uploads/2014/05/ Alianza-P\%C3\%83\%C2\%BAblico-Privada-OBEIMAYO.pdf

Servín, M. B. (2011). Nuevo Instrumento para la Atracción de Inversiones en el Paraguay. Recuperado de https://idl-bnc-idrc.dspacedirect.org/bitstream/ handle $/ 10625 / 47108 / 133483$.pdf? sequence $=1$ \&isAllowed $=y$

Stevia Market-Global Industry Analysis, Size and Forecast, 2014 to 2020 (s. f.). Recuperado el 4 de febrero de 2016, a partir de http://www. futuremarketinsights.com/reports/global-stevia-market 
Yescombe, E. R. (2007). Public-Private Partnerships. London: Elsevier.

World Economic Forum (2015). The Global Competitiveness Report 20152016. Recuperado de http://reports.weforum.org/global-competitivenessreport-2015-2016/

(C) 2018 por los autores; licencia otorgada a la revista Estudios económicos. Este artículo es de acceso abierto y distribuido bajo los términos y condiciones de una licencia Atribución-No Comercial 3.0 Unported (CC BY-NC 3.0) de Creative Commons. Para ver una copia de esta licencia, visite http://creativecommons.org/ licenses/by-nc/3 